\title{
Die aktuelle Versorgungssituation von Patientinnen und Patienten mit seltenen Knochenerkrankungen an Universitätskliniken in Deutschland
}

\section{Current structure of care and treatment options for patients with rare bone diseases at University Hospitals in Germany}

(ㄷ)(1) (요 $\ominus$

Autoren

Tobias Schmidt ${ }^{1}$, Oliver Semler ${ }^{2}$, Ralf Oheim ${ }^{1,3}$, Corinna Grasemann ${ }^{4}$, Heide Siggelkow ${ }^{5}$, Florian Barvencik ${ }^{1,3}$

Institute

1 Institut für Osteologie und Biomechanik, Universitätsklinikum Hamburg-Eppendorf, Hamburg, Deutschland

2 Zentrum für Seltene Skeletterkrankungen im Kindes- und Jugendalter, Klinik und Poliklinik für Kinder- und Jugendmedizin, Uniklinik Köln, Köln, Deutschland

3 Martin Zeitz Centrum für Seltene Erkrankungen, Universitätsklinikum Hamburg-Eppendorf, Hamburg, Deutschland

4 Zentrum für Seltene Erkrankungen Ruhr, Klinik für Kinderund Jugendmedizin Bochum, Ruhr-Universität Bochum, Deutschland

5 Med. Versorgungszentrum Endokrinologikum Göttingen, Göttingen, Deutschland

Schlüsselwörter

Seltene Knochenerkrankungen, Orphan Drugs, Zentren für seltene Erkrankungen

Keywords

Rare bone diseases, orphan drugs, centers for rare diseases, patient empowerment

Bibliografie

Osteologie 2020; 29: 266-274

DOI 10.1055/a-1233-9780

ISSN 1019-1291

(c) 2020. The Author(s).

This is an open access article published by Thieme under the terms of the Creative Commons Attribution-NonDerivative-NonCommercial-License, permitting copying and reproduction so long as the original work is given appropriate credit. Contents may not be used for commercial purposes, or adapted, remixed, transformed or built upon.

(https://creativecommons.org/licenses/by-nc-nd/4.0/)

Korrespondenzadresse

Univ.-Prof. Prof. Dr. Florian Barvencik

Institut für Osteologie und Biomechanik, Universitätsklinikum

Hamburg-Eppendorf

Lottestraße 59

\author{
22529 Hamburg \\ Germany \\ Tel.: +49-40-7410-56373 \\ E-Mail: fbarvencik@uke.de
}

\section{ZUSAMMENFASSUNG}

„Seltene Knochenerkrankungen“ ist ein Sammelbegriff für Erkrankungen des Knochenstoffwechsels, der Knochenmineralisierung sowie spezielle dysplastische Erkrankungen. An der Behandlung von betroffenen Patientinnen und Patienten sind häufig eine Vielzahl unterschiedlicher Fachdisziplinen wie Endokrinologie, Nephrologie, Orthopädie/Unfallchirurgie, Rheumatologie, Radiologie, Kardiologie, Neurologie, Innere Medizin, Pädiatrie und klinische Genetik beteiligt. Vor diesem Hintergrund ist die lokale Organisation und Steuerung der Versorgung von zentraler Bedeutung. Ziel der durchgeführten und hier vorgestellten Umfrage war es, die Angebote, Organisation und vorhandene Expertise an Universitätskliniken im Zusammenhang mit seltenen Knochenerkrankungen zu identifizieren und zu charakterisieren. 2018 wurden alle bekannten 32 Zentren für seltene Erkrankungen (ZSE) an deutschen Universitätskliniken angeschrieben. Insgesamt nahmen 15 Zentren an der Umfrage teil, die Rücklaufquote betrug somit 46,88 \%. Es zeigen sich große Unterschiede in Bezug auf das Ausrüstungsniveau und die Diagnosemöglichkeiten, die für die Behandlung von seltenen Knochenerkrankungen erforderlich sind. In den meisten Zentren sind Verfahren zur Förderung der Gesundheitskompetenz der Patientinnen und Patienten implementiert. Nur wenige Zentren sind an Forschungsaktivitäten zu seltenen Knochenerkrankungen beteiligt und bieten strukturierte Programme zur Ausbildung und zum Austausch von Fachwissen im Zusammenhang mit seltenen Knochenerkrankungen an.

Die Ergebnisse dieser Umfrage können genutzt werden, um konkrete Aktivitäten zur Verbesserung der Gesundheitsversorgung für Patientinnen und Patienten mit seltenen Knochenerkrankungen in Deutschland zu initiieren. 


\section{ABSTRACT}

Medical care for patients with Rare Bone and Mineral conditions (RBMC) require lifelong, multidisciplinary efforts including different subspecialties such as endocrinology, nephrology, orthopaedics, rheumatology, radiology, cardiology, neurology, internal medicine, paediatrics and clinical genetics. National networking and local organization and management of care in RBMC is of central importance and was assessed by means of a national survey. The aim of the study was to identify and characterize the services, organisation and existing expertise concerning RBMC at University Hospitals in Germany. All 32 Centers for Rare Diseases (ZSE) at German University Hospitals were contacted with a questionnaire in 2018. Of those, 15 centers took part in the survey, resulting in a response rate of $47 \%$. The responses revealed differences most pronounced in the level of equipment and diagnostic capabilities. While most centers had implemented tools for patient empowerment, only few centers were involved in research activities in RBMC or offered structured training programs for physicians and medical students.

The results of the survey can be helpful to initiate concrete action plans to improve healthcare for patients with RBMC in Germany.

\section{Einleitung}

In der Europäischen Union wird eine seltene Krankheit als Erkrankung mit einer Prävalenz von kleiner als 5/10000 Patientinnen und Patienten definiert [1]. Da es jedoch eine große Anzahl an seltenen Erkrankungen gibt, ist die Gesamtanzahl der Patientinnen und Patienten hoch und stellt eine große Herausforderung für das Gesundheitssystem dar. Aktuelle Schätzungen gehen von 5000-8000 seltenen Erkrankungen in Deutschland aus [2]. Hiervon sind in Deutschland schätzungsweise vier Millionen Menschen betroffen [3].

Die Diagnosestellung und Therapie von seltenen Erkrankungen ist häufig schwierig und für betroffene Patientinnen und Patienten oft mit einer langen Odyssee verbunden. Mit > $80 \%$ sind seltene Erkrankungen zudem überwiegend genetisch bedingt oder mitbedingt, manifestieren sich früh und sind in der Regel nicht heilbar. Die Seltenheit der einzelnen Erkrankungen, das fehlende Expertenwissen, fehlende kausale Therapiemöglichkeiten und erschwerte Forschungsbedingungen haben im Jahr 2010 zur Gründung des Nationalen Aktionsbündnisses für Menschen mit seltenen Erkrankungen (NAMSE) geführt [4]. NAMSE hat sich zum Ziel gesetzt, in Deutschland die Versorgungssituation für seltene Erkrankungen zu verbessern. Mit NAMSE erfolgten eine Bündelung der Initiativen im Bereich seltener Erkrankungen und die Entwicklung des nationalen Aktionsplans für Menschen mit seltenen Erkrankungen [5]. Ein zentraler Pfeiler der Aktivitäten war die Bildung und Vernetzung von Zentren zur sektorenübergreifenden Versorgung. Inzwischen haben sich insgesamt 32 sogenannte „Zentren für Seltene Erkrankungen“ (ZSE) an Universitätskliniken in Deutschland gegründet, was zu einer deutlichen Verbesserung der Versorgungssituation geführt hat. Betroffene Patientinnen und Patienten, bei denen der Verdacht auf eine seltene Erkrankung vorliegt oder eine solche diagnostiziert wird, haben in den Zentren eine regionale Anlaufstelle für weitere intradisziplinäre Diagnostik und Beratung und damit auch Zugang zu den aktuell verfügbaren Therapiemöglichkeiten.

Der Begriff „Seltene Knochenerkrankungen“ beinhaltet unterschiedliche Erkrankungen, die zu einer Beeinträchtigung z. B. von Knochenform, -struktur, -qualität, -quantität und/oder -elastizität führen. Hierzu gehören Erkrankungen des Calciumund Phosphatstoffwechsels, spezifische Störungen der Osteoblasten- oder Osteoklastenfunktion, dysplastische Erkrankungen, die zu Körperformveränderungen führen und Störungen der Mineralisation [6]. Ein Großteil der Seltenen Knochenerkrankungen sind genetisch bedingt und treten bereits im Kindesund Jugendalter durch Frakturen, Störungen des Längenwachstums oder Deformitäten in Erscheinung. In den letzten Jahren haben sich bedeutende Fortschritte in der Diagnostik und Therapie von Seltenen Knochenerkrankungen ergeben. Erkrankungen wie die Osteogensis Imperfecta (OI) und die Hypophosphatasie (HPP) können anhand von Laboruntersuchungen sowie genetischen Tests sicher diagnostiziert werden $[7,8]$. Bei der Hypophosphatasie, bei der ein Gendefekt in der alkalischen Phosphatase vorliegt, steht seit 2015 eine Enzymersatztherapie für schwer betroffene Patientinnen und Patienten mit Beginn der Erkrankung im Kindes- und Jugendalter zur Verfügung [9]. Voraussetzung für eine zielgerichtete Diagnostik und Therapie Seltener Knochenerkrankungen ist eine ausreichende Expertise in einem Zentrum, welches eine interdisziplinäre Therapie über die Lebensspanne der Patientinnen und Patienten ermöglicht. Diese Zentren sind als Fachzentren (sog. Typ-B-Zentren) den universitären Zentren für Seltene Erkrankungen (ZSE) zugeordnet.

Ziel dieser Umfrage war es, den aktuellen Versorgungsstand von Patientinnen und Patienten mit Seltenen Knochenerkrankungen in den universitären ZSE zu erfassen und Möglichkeiten der Verbesserung aufzuzeigen.

\section{Methoden}

Ein Fragebogen mit dem Briefkopf der deutschen Gesellschaft für Osteologie (DGO) mit Fragen zu Basisinformationen (Institution, Fachrichtung, Anzahl an behandelten Patientinnen und Patienten, Anteil an Minderjährigen) sowie speziellen Fragen zur Patientenedukation, Organisation und Patientenmanagement, Lehrausbildung, zum Austausch von Informationen, zu Datenbanken, epidemiologischen Studien, Qualitätssicherung und Patientensicherheit sowie Forschung wurde an alle 32 gelisteten universitären Zentren für Seltene Erkrankungen verschickt [10] ( Abb. 1). Als Kontaktadresse wurde die angegebene Anschrift des Zentrums auf der Homepage verwendet. Die Umfrage bestand insgesamt aus 52 Fragen. Die Teilnehmer wurden zudem um eigene Anmerkungen und Kommentare gebeten. Die Antworten erfolgten anonymisiert und mit Rückversand durch einen beigefügten frankierten 


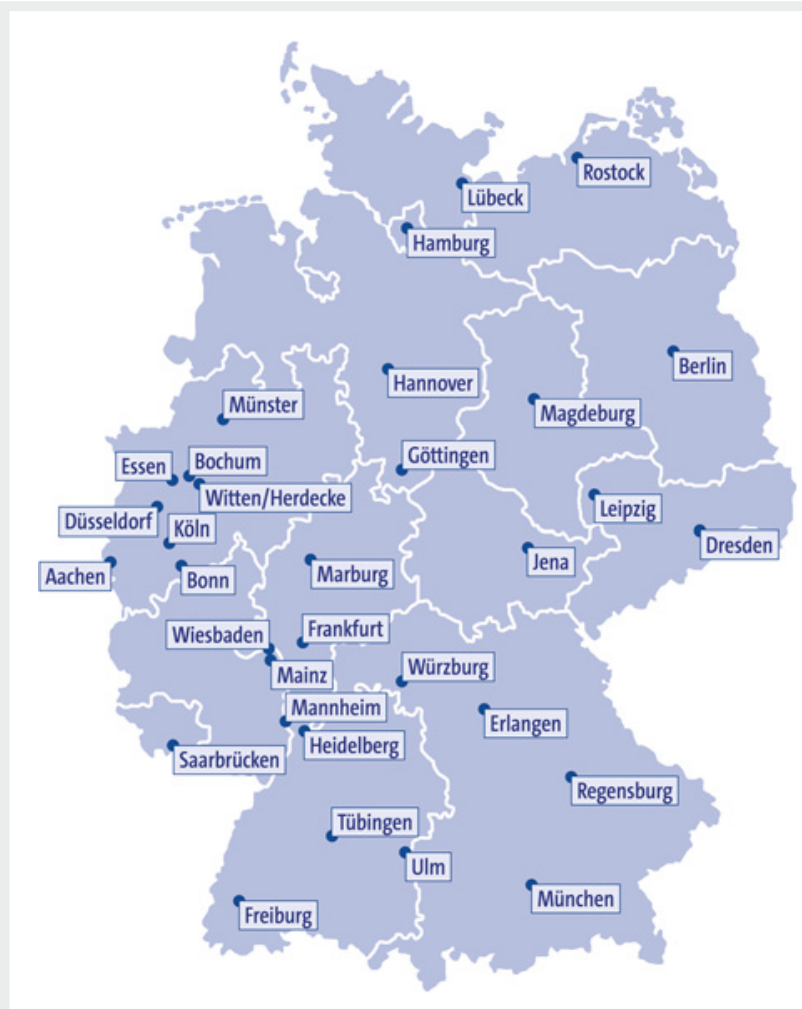

-Abb. 1 Regionale Verteilung der Zentren für Seltene Erkrankungen in Deutschland. Quelle: UKE Foto-/Grafikabteilung.

Fig. 1 Regional distribution of centres for rare diseases in Germany. Source: UKE Foto-/Grafikabteilung.

Briefumschlag. Die Ergebnisse wurden als Summe der positiven Antworten für jedes Level (vollständig umgesetzt, teilweise umgesetzt, nicht umgesetzt) ausgewertet.

\section{Ergebnisse}

Der Fragebogen wurde an alle 32 ZSE ( $\triangleright$ Abb. 1) in Deutschland verschickt. Insgesamt betrug die Rücklaufquote 46,88\% (15/32). $47 \%$ der Befragten gaben hierbei an, Facharzt für Innere Medizin zu sein, gefolgt von Pädiatrie und Orthopädie/Unfallchirurgie ( Abb. 2A). Bei der Anzahl an jährlich behandelten Patientinnen und Patienten bestanden große Unterschiede. Hier gaben $33 \%$ der Befragten an, 10-25 Patientinnen und Patienten mit Seltenen Knochenerkrankungen jährlich zu behandeln, während jeweils 20\% 50-100 und 100-500 Patientinnen und Patienten im Jahr sehen ( $\triangleright$ Abb. 2B). Der Anteil an Kindern und Jugendlichen lag bei der Hälfte der Rückmeldungen (50\%) bei 0-10\% der Patientinnen und Patienten, bei weiteren $20 \%$ bei $75-100 \%$ ( $\triangleright$ Abb. 2C). Eine PubMed-gelistete Publikation zu Patientinnen und Patienten mit Seltenen Knochenerkrankungen in den letzten Jahren gaben $53 \%$ der 15 Zentren an, wobei es große Unterschiede in der Anzahl der Publikationen gab ( $\triangleright$ Abb. 2D).

Während die Mehrzahl der 15 Zentren einen Anteil von 0$10 \%$ an dysplastischen Erkrankungen in ihrem Kollektiv angaben, werden deutlich mehr Betroffene mit Erkrankungen des Calciumund Phosphatstoffwechsels sowie mit Erkrankungen der

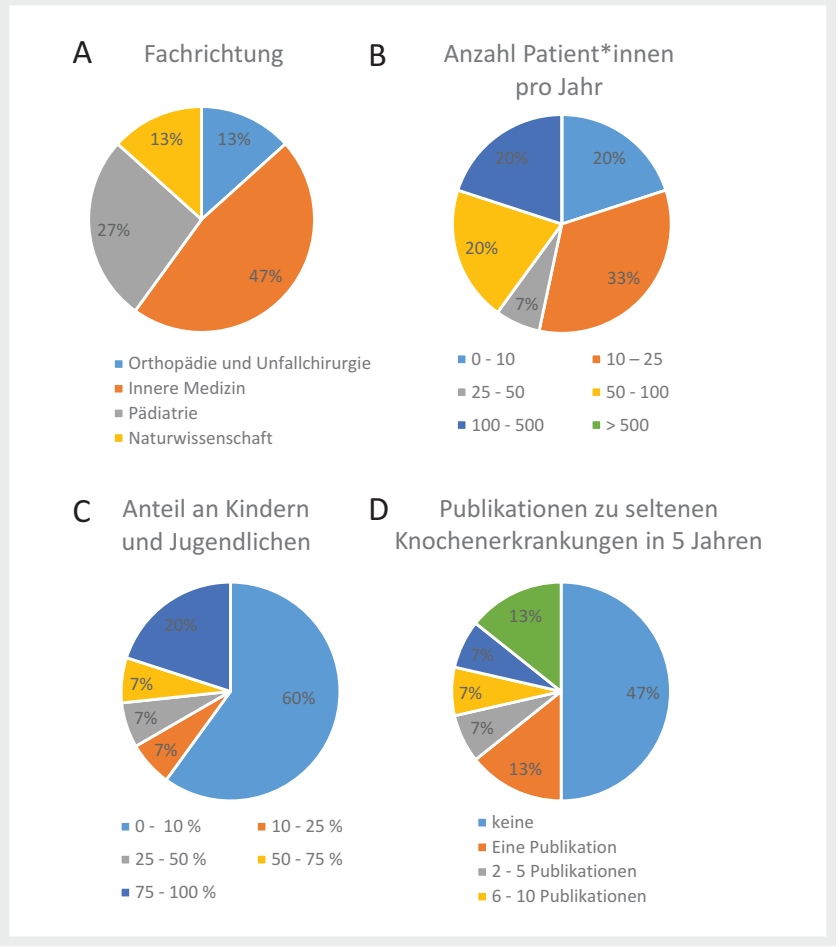

Abb. 2 Klinische Charakteristiken der Expertise für Seltene Knochenerkrankungen mit Verteilung der (A) Fachrichtung, (B) Anzahl an Patientinnen und Patienten, (C) Anteil an Kinder und Jugendlichen und (D) Publikationen zu Seltenen Knochenerkrankungen in den letzten 5 Jahren. Verteilung in Prozent der 15 Zentren mit Rückantwort dargestellt.

-Fig. 2 Clinical characteristics of expertise in rare bone diseases with distribution of (A) specialty, (B) number of patients, (C) proportion of children and adolescents, and (D) publications on rare bone diseases in the last 5 years. Distribution are shown as percentages of the centers with a response.
Knochenstruktur behandelt ( $\triangleright$ Abb. $3 A-C$ ). Die am häufigsten behandelten Erkrankungen (hierfür wurde im Fragebogen um jeweils 5 Angaben gebeten) wurden in absteigender Reihenfolge Osteogenesis imperfecta, Fibrodysplasia ossificans progressiva, Hypophosphatasie, Morbus Paget, primärer Hyperparathyreoidismus, X-chromosomale Hypophosphatämie, Chondroplasie, onkogene Osteomalazie, renale Osteopathie, Osteopetrose und chronisch rekurrierende multifokale Osteomyelitis (CRMO) genannt ( $\triangleright$ Abb. 3D).

Bei den Antworten zur Implementierung von Patientenedukation gaben die Mehrzahl der 15 Zentren an, dass transparente Behandlungsrichtlinien umgesetzt und nationale und europäische Standards zum Umgang mit Patientenrechten und Datenschutz beachtet werden. Eine Aufklärung von Patientinnen und Patienten über die eigene Erkrankung mittels Flyer und Broschüren ist nur teilweise etabliert ( $\triangleright$ Abb. 4A).

Bezüglich der Organisationstruktur der Patientenversorgung gaben alle 15 Zentren an, in unmittelbarer Nähe Zugang zu einer Intensivstation, Notaufnahme und zu Operationsmöglichkeiten zu haben. Von allen Zentren wurde die Möglichkeit zu 


\section{A}

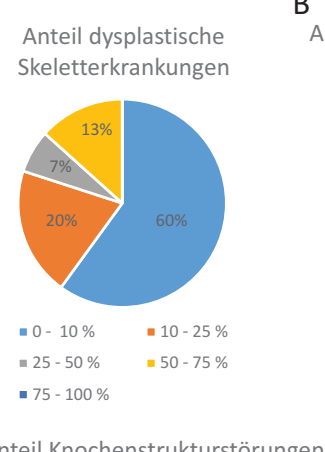

Anteil Knochenstrukturstörungen

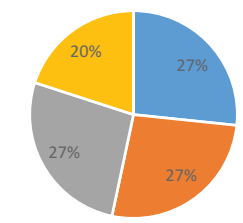

- $0-10 \%=10-25 \%=25-50 \%$

$=50-75 \% \quad-75-100 \%$
Anteil Erkrankungen des Calcium,Phosphatstoffwechsels

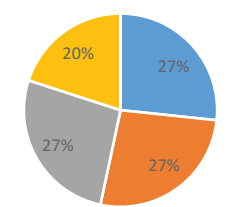

- $0-10 \% \quad-10-25 \%$

- $25-50 \%=50-75 \%$

- $75-100 \%$

D

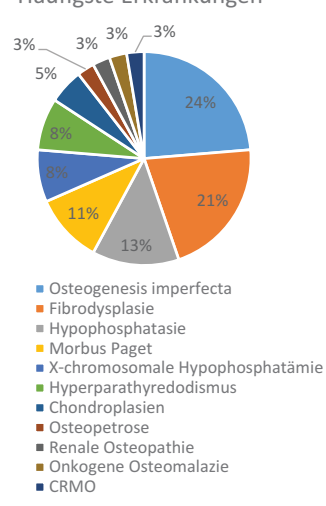

Abb. 3 Klinische Charakteristiken der Expertise für Seltene Knochenerkrankungen mit Verteilung des (A) Anteils an dysplastischen Skeletterkrankungen, des (B) Anteils an Erkrankungen des Calciumund Phosphatstoffwechsel, des (C) Anteils an Erkrankungen der Knochenstruktur und (D) am häufigsten behandelten Erkrankungen. Verteilung in Prozent der 15 Zentren mit Rückantwort dargestellt.

- Fig. 3 Clinical characteristics of expertise in rare bone diseases with distribution of (A) proportion of dysplastic skeletal diseases, (B) proportion of calcium and phosphate metabolism diseases, (C) proportion of bone structure diseases and (D) most frequently treated diseases. Distribution are shown as percentages of the centers with a response.

Laboruntersuchungen mit Parametern des Knochenstoffwechsels sowie zu humangenetischen Untersuchungen angegeben.

Ein Entlassungsmanagement ist bei ca. $50 \%$ der Befragten etabliert. Eine standardisierte Erfassung des Schmerzniveaus wird bei 80 \% teilweise oder vollständig durchgeführt, eine Quantifizierung der Einschränkung im Alltag (SF-36) teilweise bei 70 \% der Befragten ( $\triangleright$ Abb. 4B).

Bei Fragen zur Lehrausbildung zeigte sich, dass 10 von 15 Zentren regelmäßig an Fortbildungen zur Behandlung von Seltenen Knochenerkrankungen teilnehmen und 9 von 15 Zentren auch Fortbildungen veranstalten ( $\triangleright$ Abb. 5A). 10 der 15 Zentren gaben an, im Rahmen der akademischen Ausbildung Lehrveranstaltungen zu Seltenen Knochenerkrankungen durchzuführen.

Eine standardisierte Verwendung des ICD-10-Systems ist in der Behandlung der Patientinnen und Patienten in $90 \%$ der Zentren implementiert, und bei $80 \%$ erfolgt ein regelmäßiger Austausch mit anderen Zentren zur Behandlung von Patientinnen und Patienten mit Seltenen Erkrankungen. Bei über $50 \%$ der Zentren werden vollständig elektronische Medien wie Patientemsoftware und Telemedizin eingesetzt ( $\triangleright$ Abb. 5B).

Während sich nur 6 von 10 Zentren an EU-weiten Kollaborationen wie EU-Datenbanken beteiligen, wird in 12 Zentren teilweise oder vollständig eine lokale Datenbank verwendet. 9 Zentren nehmen an einer nationalen Registerstudie teil ( Abb. 6A).

In Angaben zur Evaluation von Patientenzufriedenheit und Qualitätssicherung gaben fast 50 \% der Zentren an, dass regelmäßig eine Evaluation der Produktivität des Zentrums durchgeführt wird, aber nur 2 Zentren gaben an, die Effektivität und die Qualität von Patientenschulungen zu evaluieren. Eine teilweise oder vollständige Implementation zur Erfassung der Patientenzufriedenheit wurde von $60 \%$ der Zentren angegeben (

Ein Großteil der befragten Zentren gab an, dass grundsätzlich Forschungsaktivitäten am Zentrum durchgeführt werden und hierzu Zugang besteht, allerdings gaben ca. $50 \%$ an, keine klinische oder Grundlagenforschung zu Seltenen Knochenerkrankungen durchzuführen ( $\triangleright$ Abb. 7). Somit lag die Angabe einer PubMed-gelisteten Publikation innerhalb der letzten 5 Jahre bei ca. $50 \%$. Eine Einbindung in EU-weite oder nationale Forschungskollaboration besteht bei ca. $25 \%$ der Zentren.

\section{Diskussion}

Die Ergebnisse dieser Studie sollen die Stärken und Schwächen in der Versorgung von Patientinnen und Patienten mit Seltenen Knochenerkrankungen in Deutschland charakterisieren. Hierfür wurden bewusst einige Limitationen in Kauf genommen. Da das nationale Aktionsbündnis für Menschen mit Seltenen Erkrankungen (NAMSE) zur Gründung von 32 Zentren für Seltenen Erkrankungen an deutschen Universitätskliniken geführt hat [4, 5], waren diese Zentren Anlaufstelle der durchgeführten Untersuchung. Die Etablierung von Zentren stellt einen wichtigen Schritt in der Verbesserung der Versorgungssituation für Patientinnen und Patienten mit seltenen Erkrankungen dar. Besonders die diagnostischen Möglichkeiten und interdisziplinären Therapieoptionen übersteigen bei weitem die Möglichkeiten in der ambulanten Versorgung. Dennoch muss bei der Interpretation der dargestellten Ergebnisse berücksichtigt werden, dass auch außerhalb der universitären Zentren für Seltene Erkrankungen an einigen Standorten eine exzellente medizinische Expertise für Patientinnen und Patienten mit Seltenen Knochenerkrankungen zur Verfügung steht, welche in dieser Untersuchung nicht abgebildet ist. Eine weitere Limitation stellt die Rückläuferquote von nur $47 \%$ (15 Zentren von 32 Zentren) dar. Es bleibt daher unklar, ob in den 17 Zentren ohne Rückantwort keine Expertise bzw. Struktur für Patientinnen und Patienten mit Seltenen Knochenerkrankungen vorhanden ist oder ob diese im gleichen Maße oder sogar besser dargestellt ist als in den Zentren mit Rückantwort.

Anhand der vorliegenden Ergebnisse lässt sich feststellen, dass nur eine kleine Anzahl an Zentren mehr als 100 Patientinnen und Patienten mit Seltenen Knochenerkrankungen behandelt. Der Großteil der Zentren behandelt ca. 10-25 Patientinnen und Patienten im Jahr. Dies lässt die Schlussfolgerung zu, dass aktuell nur ein kleiner Anteil der Betroffenen mit einer Seltenen 
Setzen Sie in Ihrem Zentrum transparente Behandlungsrichtlinien um (Aufzeigen von Behandlungsalternativen, Studienergebnisse, Qualitätsstandards)?

Stellen Sie sicher, dass eine informierte Zustimmung (informed consent) der Patient*innen zur Behandlung gegeben wird?

Werden nationale und europäische Standards des Umgangs mit Patientenrechten und Datenschutz umgesetzt?

Gibt es in Ihrem Zentrum einen geregelten Mechanismus der Erfassung von Patientenerfahrungen (Feedback-Bögen etc.)?

Sind in Ihrem Zentrum Maßnahmen ergriffen worden, um die Aufklärung von Patient*innen über die eigene Erkrankung zu unterstützen (Flyer, Broschüren, etc.)?

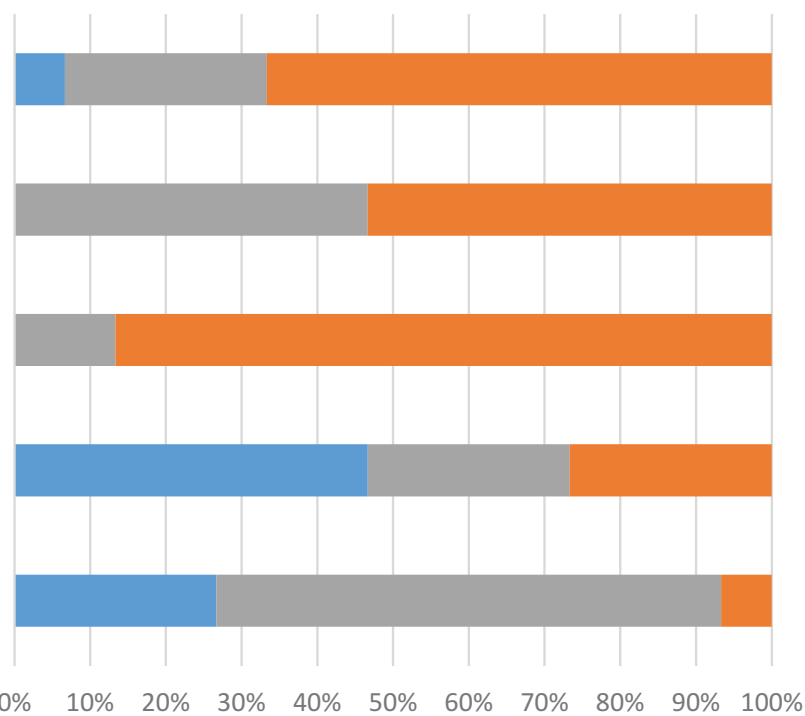

घ Nicht umgesetzt $\quad$ Teilweise umgesetzt $\quad$ Vollständig umgesetzt

B

Patientenorganisation

Besteht die Möglichkeit auf einfache Weise auf andere Betriebsmittel oder Hilfsquellen zurückzugreifen?

Gibt es in Ihrem Zentrum ein strukturiertes Programm mit einen Koordinator und einer Unternehmensführung, um Tätigkeiten und Berichte zu koordinieren?

Findet bei Ihnen eine standardisierte Erfassung der Einschränkungen von Patient*innen im Alltag (z.B. SF-36) statt? (nur für erwachsene PatientInnen)

Findet bei Ihnen eine standardisierte Erfassung des Schmerzniveaus (z.B. VAS, Brief Pain Inventory) von Patient*innen statt?

Besteht ein Programm zur regelmäßigen Anbindung der Patient*innen nach Entlassung?

Stehen bei Ihnen im Zentrum folgende Versorgungseinheiten zur Verfügung: OP, Intensivstation, Notaufnahme, Humangenetik?

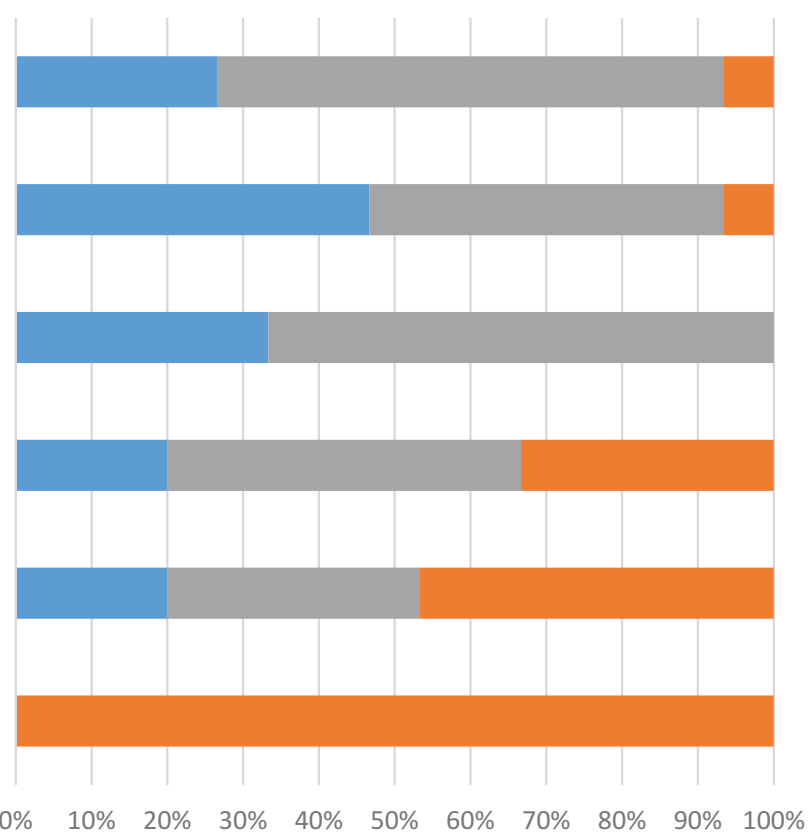

nicht umgesetzt $\quad$ Teilweise umgesetzt $\quad$ Vollständig umgesetzt

- Abb. 4 Prozentuale Verteilung für spezifische Fragen zur (A) Patientenedukation und (B) Patientenorganisation. Verteilung in Prozent der 15 Zentren mit Rückantwort, dargestellt mit blau = nicht umgesetzt, grau = teilweise umgesetzt und orange = vollständig umgesetzt.

- Fig. 4 Percentage distribution for specific questions on (A) patient education and (B) patient organization. Distribution are shown in percent of the 15 centers with responses with; blue = not implemented, gray = partially implemented and orange = fully implemented. 
A

\section{Lehrausbildung}

Nehmen Sie regelmäßig an Fortbildungen zur Behandlung von seltenen Knochenerkrankungen teil?

Veranstalten Sie Fortbildungen zur Behandlung von seltenen Knochenerkrankungen außerhalb Ihres Zentrums?

Finden bei Ihnen im Zentrum Fortbildungen zur Behandlung von seltenen Knochenerkrankungen statt?

Findet bei Ihnen im Zentrum eine akademische Ausbildung zur Behandlung von Patient*innen mit seltenen Knochenerkrankungen statt?

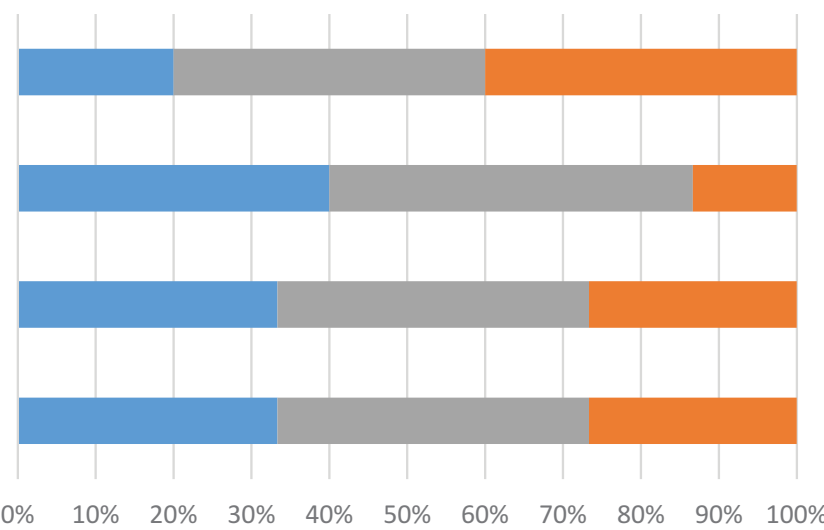

घicht umgesetzt $\quad$ Teilweise umgesetzt $\quad$ Vollständig umgesetzt

B

\section{Austausch von Informationen}

Werden bei Ihnen standardisierte Codiersysteme (ICD-10) verwendet?

Werden bei Ihnen im Zentrum regelmäßig elektronische Systeme eingesetzt (Patientensoftware, Telemedizin, etc.)

Gibt es standardisierte Prozeduren, um einen sicheren Austausch von medizinischen Daten zu erlauben?

Besteht die Möglichkeit eines regelmäßigen Austausches mit anderen Zentren zur Behandlung von seltenen Knochenerkrankungen?

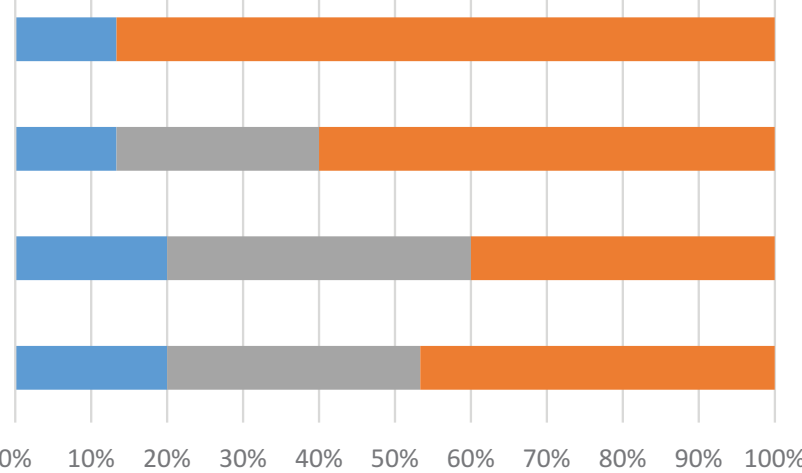

Nicht umgesetzt

- Teilweise umgesetzt

- Vollständig umgesetzt

Abb. 5 Prozentuale Verteilung für spezifische Fragen zur (A) Lehrausbildung und (B) Austausch von Informationen. Verteilung in Prozent der 15 Zentren mit Rückantwort, dargestellt mit blau = nicht umgesetzt, grau = teilweise umgesetzt und orange = vollständig umgesetzt.

- Fig. 5 Percentage distribution for specific questions on (A) apprenticeship training and (B) exchange of information. Distribution are shown in percent of the 15 centers with responses with; blue = not implemented, gray = partially implemented and orange = fully implemented.

Knochenerkrankung in Deutschland in einem Zentrum für Seltene Knochenerkrankungen behandelt wird. Der Vorteil der Bündelung von Erkrankungen an Zentren mit spezifischer Expertise liegt vor allem in der schnelleren Diagnose und frühzeitigen Therapieeinleitung. Um die verfügbaren Zentren für eine größere Anzahl von Betroffenen sichtbar zu machen, wäre eine stärkere Bekanntmachung der Möglichkeiten der universitären Zentren für den ambulanten Bereich ein sinnvoller Ansatz. Die Zunahme der Zahl von Betroffenen an den Zentren würde dann die Entwicklung der hochspezialisierten Zentren an den Universitätskliniken weiter unterstützen.

Die Wichtigkeit der frühzeitigen Diagnose und fachgerechten Therapie hat sich in den letzten Jahren durch die Etablierung neuer Therapieoptionen für einige Seltene Knochenerkrankungen noch erhöht. So steht für schwer betroffene Patientinnen und Patienten mit einer im Kindes- und Jugendalter diagnostizierten Hypophosphatasie eine hochwirksame Enzymersatztherapie zur Verfügung [7], eine Osteogensis imperfecta kann mit intravenösen Bisphosphonaten behandelt werden [11], ein FGF-23-Antikörper kann zur Therapie der X-chromosomalen Hypophosphatämie im Kindesalter eingesetzt werden [12], und Betroffene mit Hypoparathyreoidismus, die unter konventioneller Therapie nicht ausreichend gut eingestellt sind, können mit rekombinantem PTH 1-84 behandelt werden [13]. Es macht Sinn, dass diese Therapien und das Therapiemonitoring durch mit dem Krankheitsbild und der Behandlung erfahrene Expertinnen und Experten 
A

\section{Datenbanken}

Nehmen Sie an EU-weiten Kollaborationen (z.B. EU Datenbanken) teil?

Waren Sie in den letzten 5 Jahren Prüfarzt einer Studie zu seltenen Knochenerkrankungen?

Haben Sie in den letzten 5 Jahren epidemiologische Studien durchgeführt und diese publiziert?

Nehmen sie an einer nationalen Registerstudie mit verschlüsselten Patienten IDs teil?

Gibt es bei Ihnen im Zentrum eine lokale Datenbank mit einer verschlüsselten Patienten ID?

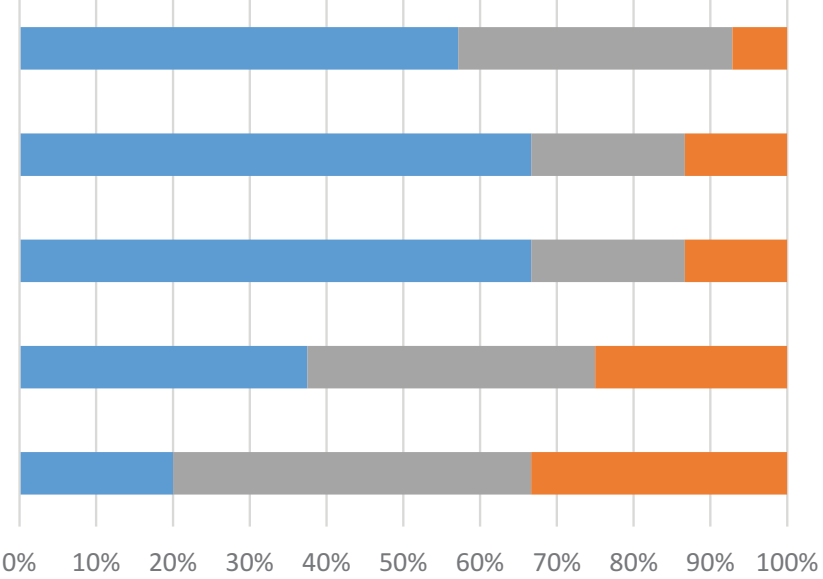

Nicht umgesetzt $\quad$ Teilweise umgesetzt $\quad$ Vollständig umgesetzt

B

Entwickeln und nutzen Sie klinische Leitlinien zur Behandlung von seltenen Knochenerkrankungen?

Findet eine regelmäßige Evaluation der Produktivität des Zentrums (Publikationen, Patientenzahlen, etc.) statt?

Findet eine regelmäßige Evaluation des medizinischen Personals statt?

Gibt es einen Plan zur standardisierten Erfassung der Effektivität und Qualität von Patientenschulungen?

Gibt es bei Ihnen im Zentrum ein standardisiertes Management (z.B. Feedback-Bögen) zur Erfassung der Patientenzufriedenheit?

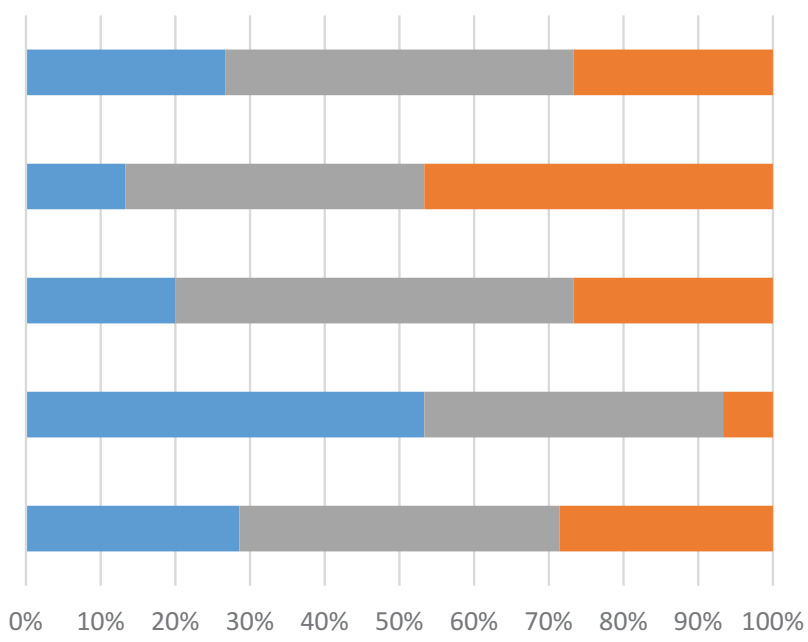

$\begin{array}{lllllllllll}0 \% & 10 \% & 20 \% & 30 \% & 40 \% & 50 \% & 60 \% & 70 \% & 80 \% & 90 \% & 100 \%\end{array}$

nicht umgesetzt $\quad$ Teilweise umgesetzt $\quad$ Vollständig umgesetzt

- Abb. 6 Prozentuale Verteilung für spezifische Fragen zur (A) Datenbanken und (B) Patientenzufriedenheit. Verteilung in Prozent der 15 Zentren mit Rückantwort, dargestellt mit blau = nicht umgesetzt, grau = teilweise umgesetzt und orange = vollständig umgesetzt.

- Fig. 6 Percentage distribution for specific questions on (A) databases and (B) patient satisfaction. Distribution are shown in percent of the 15 centers with responses with; blue $=$ not implemented, gray = partially implemented and orange = completely implemented.

durchgeführt werden. Auch hier kommt den Zentren für Seltene Knochenerkrankungen eine entscheidende Rolle zu.

Die Umfrage offenbart auch deutlichen Aufholbedarf im Bereich der Forschungssituation zu Seltenen Knochenerkrankungen. Möglicherweise aufgrund der universitären Anbindung sind zwar alle Zentren in Forschungsaktivitäten eingebunden, allerdings führen nur ca. die Hälfte der sich an der Umfrage beteiligten Zentren eigenständig Forschung zu Seltenen Knochenerkrankungen durch. Auch sind an nationalen oder internationalen Datenbanken nur eine geringe Anzahl an Zentren beteiligt.

Diese Auswertung spiegelt die aktuellen Schwierigkeiten in der Forschung zu Seltenen Erkrankungen im Bereich des Knochens wider. Die Ursachen sind sicher vielschichtig, aber u.a. ist hier eine deutlich höhere finanzielle Unterstützung von Forschungsprojekten vonnöten, um für Betroffene in der Zukunft eine verbesserte Diagnostik und kausale Therapie anbieten zu können. 


\section{Forschung}

Haben Sie in den letzten 5 Jahren an einer EU-weiten Forschungskollaboration teilgenommen?

Haben Sie in den letzten 5 Jahren an einer nationalen Forschungskollaboration teilgenommen?

Nehmen sie an einem EU-weiten Programm zur Erforschung von seltenen Knochenerkrankungen teil?

Gehören Sie zu einem nationalen Programm zur Erforschung von seltenen Knochenerkrankungen?

Haben Sie in den letzten 12 Monaten in einem PubMed gelisteten Journal publiziert?

Findet in Ihrem Zentrum Grundlagenforschung zu seltenen Knochenerkrankungen statt?

Werden bei Ihnen klinischen Studien zu seltenen Knochenerkrankungen durchgeführt?

Hat Ihr Zentrum Zugang zu Forschungseinrichtungen?

Führt Ihr Zentrum Forschungsaktivitäten durch?

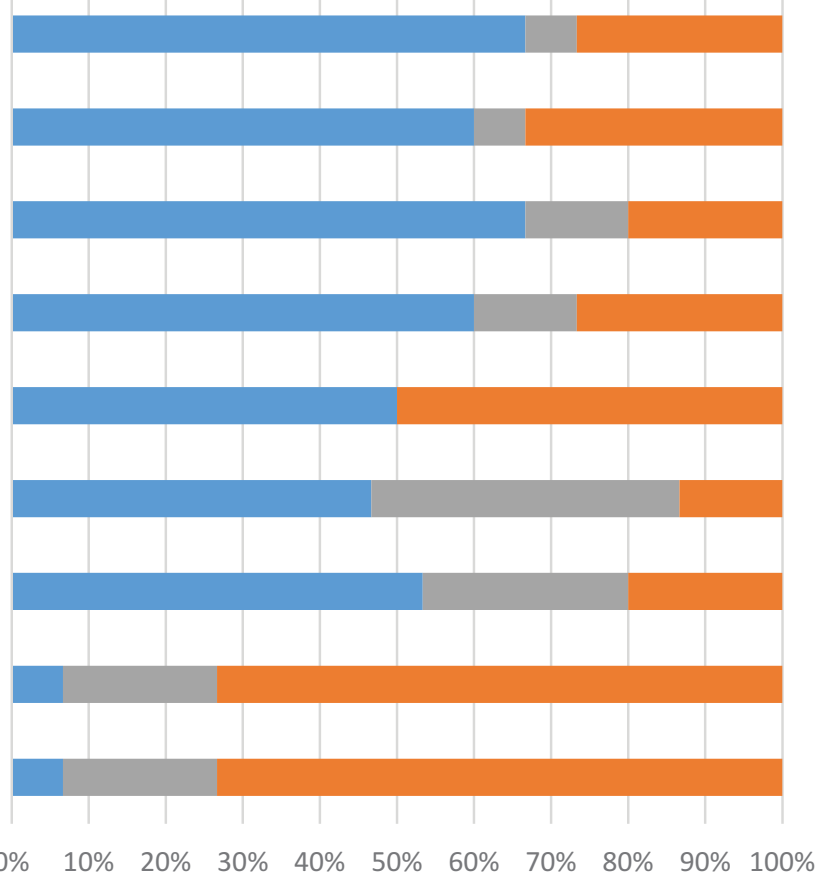

- Nicht umgesetzt $\quad$ Teilweise umgesetzt $\quad$ Vollständig umgesetzt

Abb. 7 Prozentuale Verteilung für spezifische Fragen zu Forschungsaktivitäten. Verteilung in Prozent der 15 Zentren mit Rückantwort, dargestellt mit blau = nicht umgesetzt, grau = teilweise umgesetzt und orange = vollständig umgesetzt.

- Fig. 7 Percentage distribution for specific questions on research activities. Distribution are shown in percent of the 15 centers with responses with; blue = not implemented, gray = partially implemented and orange $=$ completely implemented.

\section{Schlussfolgerung}

Diese Umfrage zeigt große Unterschiede in der Versorgungsstruktur von Patientinnen und Patienten mit Seltenen Knochenerkrankungen an deutschen Universitätskliniken. Auch wenn durch die Bildung von Zentren im Rahmen des nationalen Aktionsplans für Menschen mit Seltenen Erkrankungen ein wichtiger Schritt zur Verbesserung der Diagnose- und Therapiemöglichkeiten getan wurde, besteht weiterhin insbesondere ein Mangel an der Koordination und Organisation einer interdisziplinären Behandlung. Der Anteil an unterschiedlichen Fachdisziplinen in der Behandlung von Patientinnen und Patienten mit Seltenen Knochenerkrankungen ist divers und beinhaltet Expertise von Orthopädie, Innerer Medizin, Pädiatrie und Naturwissenschaften. Die Vorteile einer interdisziplinären Behandlung von Patientinnen und Patienten mit Seltenen Erkrankungen ist gut beschrieben, allgemein anerkannt und entscheidender Teil des europäischen Versorgungsplans [14-16]. Den Zentren kommt hierbei die wichtige Rolle zu, alle beteiligten Disziplinen zu koordinieren, um die bestmögliche Therapie für die Patientinnen und Patienten zu erreichen. Deutliches Verbesserungspotenzial besteht allerdings auch an den bestehenden Zentren, da nur 2
Zentren bisher ein durchgehend strukturiertes Programm mit einem Koordinator vorhalten. Bei der Hälfte der Zentren besteht zumindest eine teilweise Umsetzung. Die Etablierung von zentralen Koordinatoren/Spezialisten, z.B. in Form von Professuren für Seltene Erkrankungen, könnte helfen, die Aufgaben für die Zukunft besser zu lösen. Die Koordination von Diagnostik und der teilweise sehr aufwendigen und teuren Therapie von Patientinnen und Patienten mit Seltenen Knochenerkrankungen ist eine zentrale Herausforderung für die Zukunft. Über die bessere Sichtbarkeit vorhandener Zentren für Seltene Knochenerkrankungen und eine zunehmende Kooperation und Spezialisierung kann auch eine entscheidende Verbesserung in der Forschung bezüglich der Seltenen Knochenerkrankungen erwartet werden.

\section{Funding Information}

Die Open Access Publikation dieses Artikels wurde von den Firmen Alexion, Kyowa Kirin und Takeda durch die Übernahme der APC ermöglicht. Es erfolgte keinerlei Einflussnahme dieser Firmen auf die Inhalte des Artikels. 
Interessenkonflikt

Die Autorinnen/Autoren geben an, dass kein Interessenkonflikt besteht.

\section{Literatur}

[1] Verordnung (EG) Nr. 141/2000 des Europäischen Parlaments und des Rates vom 16. Dezember 1999 über Arzneimittel für seltene Leiden (https://eur-lex.europa.eu/legal-content/DE/TXT/?uri=CELEX: 32000R0141).

[2] https://www.orpha.net

[3] https://www.gesundheitsforschung-bmbf.de/files/SE_Bestandsaufnahme2014.pdf

[4] https://www.gesundheitsforschung-bmbf.de/files/nationaler_aktionsplan.pdf

[5] https://www.namse.de/seltene-erkrankungen.html

[6] https://www.iofbonehealth.org/osteoporosis-musculoskeletal-disorders/skeletal-rare-disorders

[7] Whyte MP. Hypophosphatasia - aetiology, nosology, pathogenesis, diagnosis and treatment. Nat Rev Endocrinol 2016; 12 (4): 233-246

[8] Wirth T. [Osteogenesis imperfecta]. Orthopade. 2012; 41 (9): 773-782; quiz 83-4
[9] Whyte MP, Rockman-Greenberg C, Ozono K et al. Asfotase alfa treatment improves survival for perinatal and infantile hypophosphatasia. J Clin Endocrinol Metab 2016; 101 (1): 334-342

[10] https://www.se-atlas.de/map/zse/.

[11] Trejo P, Rauch F. Osteogenesis imperfecta in children and adolescentsnew developments in diagnosis and treatment. Osteoporos Int 2016; 27 (12): 3427-3437

[12] Insogna KL, Briot K, Imel EA et al. A randomized, double-blind, Placebocontrolled, phase 3 trial evaluating the efficacy of burosumab, an AntiFGF23 antibody, in adults with X-linked hypophosphatemia: week 24 primary analysis. J Bone Miner Res 2018; 33 (8): 1383-1393

[13] Sikjaer T, Amstrup AK, Rolighed L et al. PTH(1-84) replacement therapy in hypoparathyroidism: A randomized controlled trial on pharmacokinetic and dynamic effects after 6 months of treatment. J Bone Miner Res 2013; 28 (10): 2232-2243

[14] Taruscio D, Gentile AE, Evangelista T et al. Centres of expertise and European reference networks: key issues in the field of rare diseases. The EUCERD recommendations. Blood Transfusion = Trasfusione Del Sangue. 2014; 12 Suppl 3(s621-5

[15] Moliner AM. Creating a European Union framework for actions in the field of rare diseases. Adv Exp Med Biol 2010; 686: (457-73

[16] Heon-Klin V. European reference networks for rare diseases: What is the conceptual framework? Orphanet J Rare Dis 2017; 12 (1): 137 